Draft VERSion June 15, 2021

Preprint typeset using $\mathrm{L}_{\mathrm{A}}^{\mathrm{A}} \mathrm{E} \mathrm{X}$ style emulateapj v. 5/2/11

\title{
PARTIAL REFLECTION AND TRAPPING OF A FAST-MODE WAVE IN SOLAR CORONAL ARCADE LOOPS
}

\author{
PANKAJ KUMAR ${ }^{1,2}$, D.E. INNES ${ }^{2}$ \\ ${ }^{1}$ Korea Astronomy and Space Science Institute (KASI), Daejeon, 305-348, Republic of Korea and \\ ${ }^{2}$ Max-Planck Institut für Sonnensystemforschung, 37191 Katlenburg-Lindau, Germany \\ Draft version June 15, 2021
}

\begin{abstract}
We report on the first direct observation of a fast-mode wave propagating along and perpendicular to cool $(171 \AA)$ arcade loops observed by the Solar Dynamics Observatory/Atmospheric Imaging Assembly (AIA). The wave was associated with an impulsive/compact flare, near the edge of a sunspot. The EUV wavefront expanded radially outward from the flare center and decelerated in the corona from 1060-760 $\mathrm{km} \mathrm{s}^{-1}$ within $\sim 3-4$ minute. Part of the EUV wave propagated along a large-scale arcade of cool loops and was partially reflected back to the flare site. The phase speed of the wave was about $1450 \mathrm{~km} \mathrm{~s}^{-1}$, which is interpreted as a fast-mode wave. A second overlying loop arcade, orientated perpendicular to the cool arcade, is heated and becomes visible in the AIA hot channels. These hot loops sway in time with the EUV wave, as it propagated to and fro along the lower loop arcade. We suggest that an impulsive energy release at one of the footpoints of the arcade loops causes the onset of an EUV shock wave that propagates along and perpendicular to the magnetic field.

Subject headings: Sun: flares - Sun: corona-Sun: oscillations - Sun: UV radiation
\end{abstract}

\section{INTRODUCTION}

The study of Magnetohydrodynamic (MHD) waves in the solar atmosphere is very important because they provide an indirect way to probe the solar corona via coronal seismology and may play an important role in coronal heating (Uchida 1970; Roberts et al. 1984; Nakariakov \& Verwichte 2005; Banerjee et al. 2007; De Moortel \& Nakariakov 2012).

Large-scale coronal waves are generally observed during solar eruptions. These waves were discovered by the Extreme-ultraviolet Imaging Telescope (EIT) onboard the Solar and Heliospheric Observatory (SOHO), and are sometimes referred to as EIT waves (Thompson et al. 1999). The nature of these waves is still under debate. Now most of the observations from SDO/AIA and STEREO support their wave nature. These waves can be impulsively generated either by thermal pressure produced by the flare or magnetic pressure of the CME piston (Vršnak \& Cliver 2008). Using STEREO observations, Veronig et al. (2010) reported on a domeshaped EUV wave (speed $\sim 650 \mathrm{~km} \mathrm{~s}^{-1}$ ) and interpreted it as a weakly shocked fast-mode wave. At present, there are several observational studies of propagating fast-mode waves using SDO/AIA images (e.g., Chen \& Wu 2011,Ma et al. 2011, Patsourakos \& Vourlidas 2012, Nitta et al. (2014), Liu \& Ofman 2014 and references cited therein). Furthermore, transverse kink oscillations of the loops/filaments (located away from the flare) are also observed when a fast MHD wave (i.e., global EUV wave) interacts with these structures (Asai et al. 2012; Kumar et al. 2013).

Recently, fast-mode wave trains have been discovered (during solar flares/eruptions) with SDO/AIA, propagating along funnel shaped loops with the speed of 1000-2000 $\mathrm{km} \mathrm{s}^{-1}$ (Liu et al. 2011; Shen \& Liu 2012; Kumar \& Manoharan 2013). Using Hinode/SOT obser-

pankaj@kasi.re.kr vations, Ofman \& Wang (2008) reported the first observations of transverse waves in coronal multi-threaded loops with cool plasma ejected from the chromosphere flowing along the threads. They found that the waves were nearly standing (fundamental) kink modes in some of the treads with a phase speed of $\sim 1250 \mathrm{~km} \mathrm{~s}^{-1}$, whereas the dynamics of other threads was consistent with propagating fast magnetoacoustic waves. Apart from the fast-mode wave, reflecting slow-mode wave (in arcade loops) has been recently discovered in the AIA hot channels (131 and $94 \AA$ ) (Kumar et al. 2013), which was consistent with the SOHO/SUMER Doppler-shift oscillations (T>6 MK) (Kliem et al. 2002; Wang et al. 2002, 2003a,b). However, fast-mode wave propagation along closed arcade loops, its partial reflection and trapping has not been reported so far.

In this letter, we report the first direct observation of fast-mode wave propagation (along and across arcades of loops) and its partial reflection in cool (AIA $171 \AA$ ) loops. The EUV wave caused transverse oscillation, seen in the AIA 131 and $94 \AA$ channels, of loops orientated perpendicular and above the cool loop arcade. The EUV wave was observed during an impulsive/compact flare that occurred at one of the footpoints of the cool loop system on 6 March 2014. In section 2, we present the observations and in the last section, we discuss and summarize the results.

\section{OBSERVATIONS AND RESULTS}

The Atmospheric Image Assembly (AIA; Lemen et al. 2012) onboard the Solar Dynamics Observatory (SDO) records full disk images of the Sun (field-of-view $~ 1.3$ $\left.\mathrm{R}_{\odot}\right)$ with a spatial resolution of $1.5^{\prime \prime}\left(0.6^{\prime \prime}\right.$ pixel $\left.^{-1}\right)$ and a cadence of $12 \mathrm{~s}$. For the present study, we utilized $171 \AA$ (Fe IX, with formation temperature $T \approx 0.7 \mathrm{MK}$ ), $94 \AA$ (Fe XVIII, $T \approx 6.3 \mathrm{MK}$ ), and $1600 \AA$ (C IV + cont., $T \approx 0.01 \mathrm{MK})$ images.

AR NOAA 11198 ( $\beta$ magnetic configuration, S07E64) 

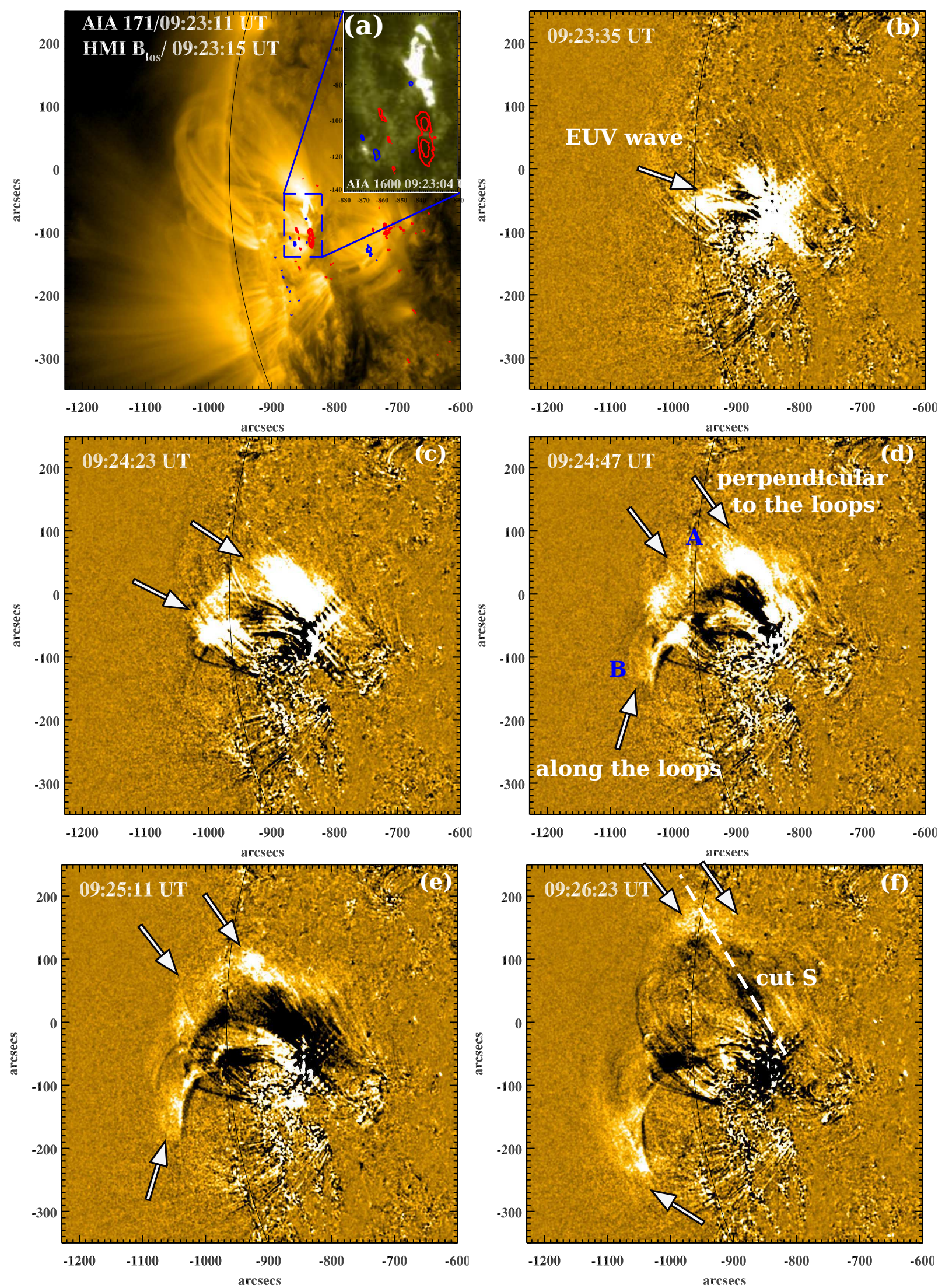

FIG. 1. - (a) HMI magnetogram contours of positive (red) and negative (blue) polarities over $171 \AA$ intensity image. The contours levels are $\pm 400, \pm 800 \mathrm{G}$. Inset shows the flaring region in $1600 \AA$ channel, which is overlaid by HMI magnetogram contours. (b-f) AIA $171 \AA$ running difference images showing the propagating EUV wave moving along and perpendicular to the loop system. (Animation is available.) 


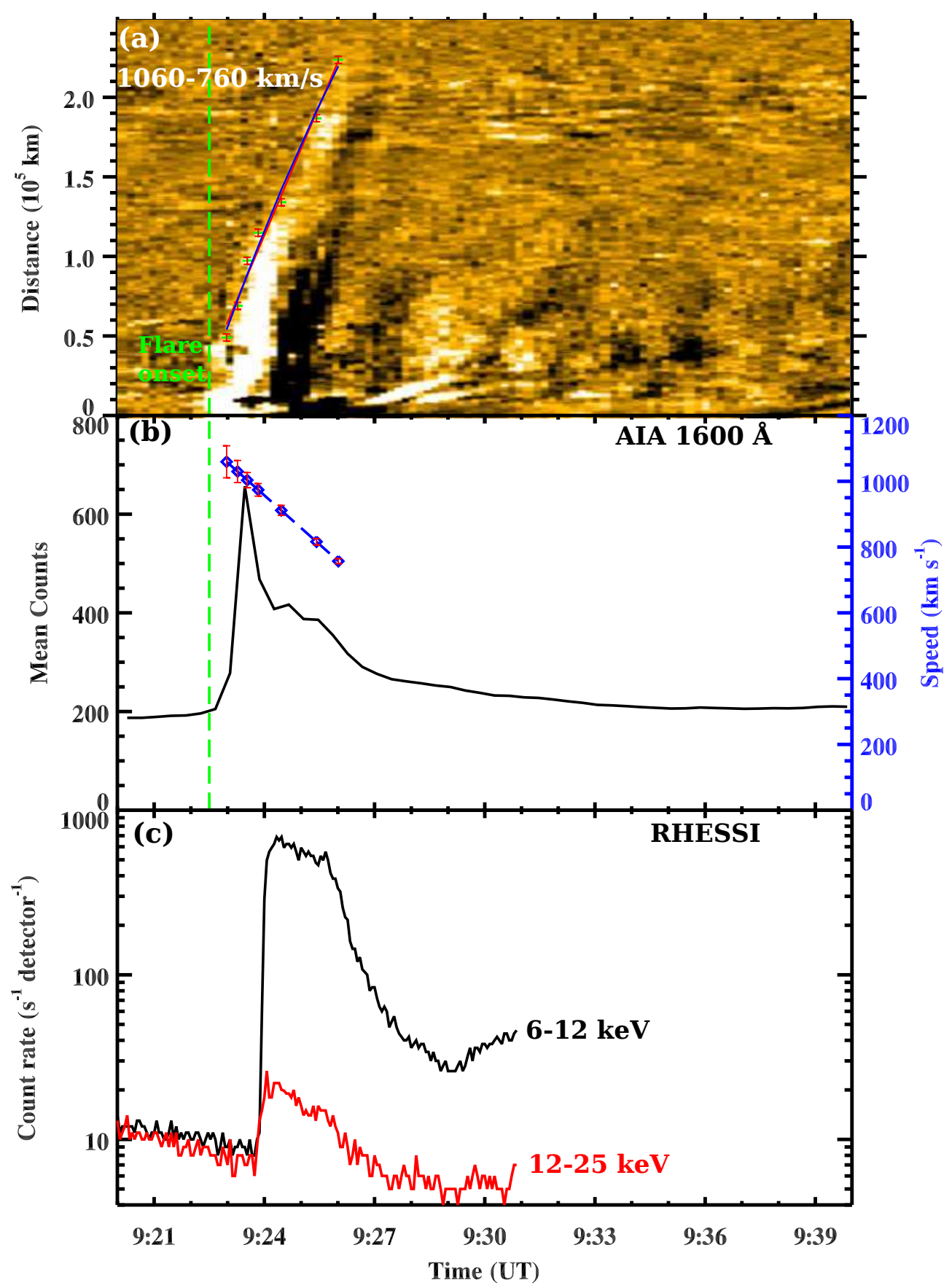

FIG. 2.- (a) Distance-time plot of the intensity distribution along the slice 'S' using $171 \AA$ running difference images. The linear fit to the data point is shown by the red curve. (b) Mean intensity profile of the flaring region, extracted using a box region at the flare site in $1600 \AA$ images. Speed profile of the EUV wave derived from a second order polynomial fitting to the wavefront in the distance-time plot (blue curve). (c) RHESSI X-ray flux profiles. Green vertical line shows the start time of the flare and associated EUV wave. 
was located on the eastern limb on 6 March 2014. The EUV wave, reported here, was associated with an impulsive/compact flare that started (in this AR) at $\sim 09: 23$ UT, maximized at $\sim 09: 25$ and ended at $\sim 09: 30 \mathrm{UT}$.

\subsection{Observation of the EUV wave}

The EUV wave is best observed in the AIA $171 \AA$ channel. We utilized AIA $171 \AA$ running difference images $(\Delta \mathrm{t}=1 \mathrm{~min})$ to study the kinematics and propagation characteristics of the EUV wave. Figure 1 displays selected AIA $171 \AA$ running difference images. We overlaid HMI magnetogram (Schou et al. 2012) contours of positive (red) and negative (blue) polarities on the AIA $171 \AA$ intensity image in the first panel (a), to show the magnetic field configuration of the flare site. The inset shows the AIA $1600 \AA$ image, exhibiting a closer view of the flare site. The brightening of a flare ribbon starts ( 09:23 UT) at the northern edge of a positive polarity sunspot. Figures 1(b) and (c) show the outward movement of the EUV disturbance/wavefront from the flare center. Figure 1(d) clearly shows the EUV wavefronts propagating perpendicular and parallel to the active region loops (09:24:27 UT). These fronts are marked by 'A' and ' $\mathrm{B}$ ', respectively. Later, we noticed that the wave reflected (front B) from the other footpoint of the arcade loops. When the returning wave reached point ' $\mathrm{B}$ ', the loops appeared to expand upwards, generating motion both outward into the corona and back to the flare site (see AIA $171 \AA$ running difference movie).

The EUV disturbance was also observed in the AIA 193 and $211 \AA$ channels, which provide better contrast for large-scale EUV waves in the solar corona (see online movie). The circular EUV wavefront shows outward expansion from the flare site. The radially outward moving front $(\mathrm{A})$ could be tracked until about 09:27 UT in the AIA field of view. To determine the kinematics of the outward moving EUV wavefront (A), we used a slice cut S (Figure 1f) to create a distance-time plot. Figure $2 \mathrm{a}$ displays the stack plot of running difference intensity along the slice $\mathrm{S}$. The wavefront is clearly observed from $\sim 09: 23$ UT to 09:26 UT. We estimated the mean speed of the wavefront, using a linear fit to the visually selected data points (red curve), to be $\sim 910 \pm 10 \mathrm{~km} \mathrm{~s}^{-1}$. We assumed 5 pixel uncertainty in the identification of the EUV wavefront. To deduce the speed profile (range) of the wavefront, we fitted a second order polynomial function (blue curve) to the observed data points and estimated the speed. Figure 2(b) shows the speed profile of the wave. The EUV wave decelerates from $\sim 1060$ to $760 \mathrm{~km} \mathrm{~s}^{-1}$ in the $3-4$ minute interval.

To examine the relationship between the EUV wave and the flare, we plotted the AIA $1600 \AA$ mean flux of the flare region as shown in Figure 1a. Figure 2(b-c) shows the AIA $1600 \AA$ flux profile and RHESSI X-ray flux profiles in the $6-12$ and $12-25 \mathrm{keV}$ energy channels. It is evident that the onset of the EUV wave and the rising of the $1600 \AA$ flux evolve simultaneously. Therefore, the trigger of the EUV wave is closely related with the flare energy release. There was no plasmoid eruption during the flare onset in any of the AIA channels (i.e., AIA 304, 131, 94 etc.).

2.2. Partial reflection and oscillation of hot loops
The onset of the EUV wave and flare energy release occurred simultaneously at $\sim 09: 23$ UT. The partial reflection of the EUV wave was observed along the arcade loops in the $171 \AA$ and $193 \AA$ channels.

Figures 3(a) and (c) show the running difference images in AIA 171 and $94 \AA$ channels, respectively. To investigate the wave propagation, we selected a path along the loop (in blue color) and extracted the 171 and $94 \AA$ running-difference intensity $(\Delta t=1 \mathrm{~min})$ during 09:20 09:40 UT. Figure 3(b) displays the stack plots of the intensity distribution along the selected loop (shown by blue dots). The AIA $171 \AA$ stack plot (panel b) reveals the propagating wave along the loop and partially reflecting EUV wave from the opposite footpoint of the arcade loops (green dashed line). The first two white arrows indicate the time of the EUV wave onset and the time of its returning (after partial reflection) (see $171 \AA$ movie). The travel time is $10 \mathrm{~min}$.

During its outward propagation, the EUV wave encountered an arcade of loops overlying the $171 \AA$ loops. These overlying loops became visible in the 94 and $131 \AA$ channels, implying that they were heated during flare onset. One footpoint was attached to the flare site (Figure $3(\mathrm{c})$ ) so the initial heating was most likely caused by energetic particles generated in the flare. We observed a single RHESSI (Lin et al. 2002) hard X-ray source (12$25 \mathrm{keV}$ ) during the flare maximum (09-24-09:25 UT). Simultaneously, we noticed impulsive footpoint heating/brightening in the AIA $1600 \AA$ channel (refer to the AIA $1600 \AA$ movie). Acceleration of nonthermal electrons possibly precipitated to the footpoint causing impulsive heating in the AIA $1600 \AA$ channel (Figure 3(e)). Figure 3(f) shows the RHESSI spectrum fitted (energy range, 6-30 keV) with isothermal ( $\mathrm{T}=15.6 \mathrm{MK}$, $\mathrm{EM}=4.5 \times 10^{46} \mathrm{~cm}^{-3}$ ) and thick-target bremsstrahlung (spectral index=5.7) components during 09:24-09:25 UT. We can see that the non-thermal contribution dominates over the thermal component above $\sim 13 \mathrm{keV}$. In addition, we observed a type III radio burst at 09:23-09:24 UT, suggesting the acceleration of non-thermal electrons upward into the interplanetary medium (Figure 5c). However, thermal contribution from the flare site may contribute to heating the loop in the later phase.

The heated loops are more visible in the AIA $94 \AA$ than in the AIA $131 \AA$ channel. The hot loops swayed in time with the EUV wave seen along the $171 \AA$ loops. Figure $3 \mathrm{~d}$ shows a stack plot (base and running difference) of the $94 \AA$ emission along the selected slice in Figure 3c. The transverse oscillation of the hot loops was triggered by the outward propagating fast-mode wave and partially reflected wave. The arcade movement $\left(\sim 312 \mathrm{~km} \mathrm{~s}^{-1}\right)$ observed in the $94 \AA$ images is consistent with the passage of the EUV wave observed in the $171 \AA$ running difference images (Figure $3 b$ ).

There is a dimming along the $171 \AA$ loops at about 09:30 UT in the running difference movies (AIA 171 and $193 \AA$ channels). The dimming is visible in Figure $3 \mathrm{~b}$. It occurred over a large region of the loops, just after the partial reflection. At the same time a $171 \AA$ front was seen descending towards the flare footpoint.

To track the direction of the waves over the regions, we 

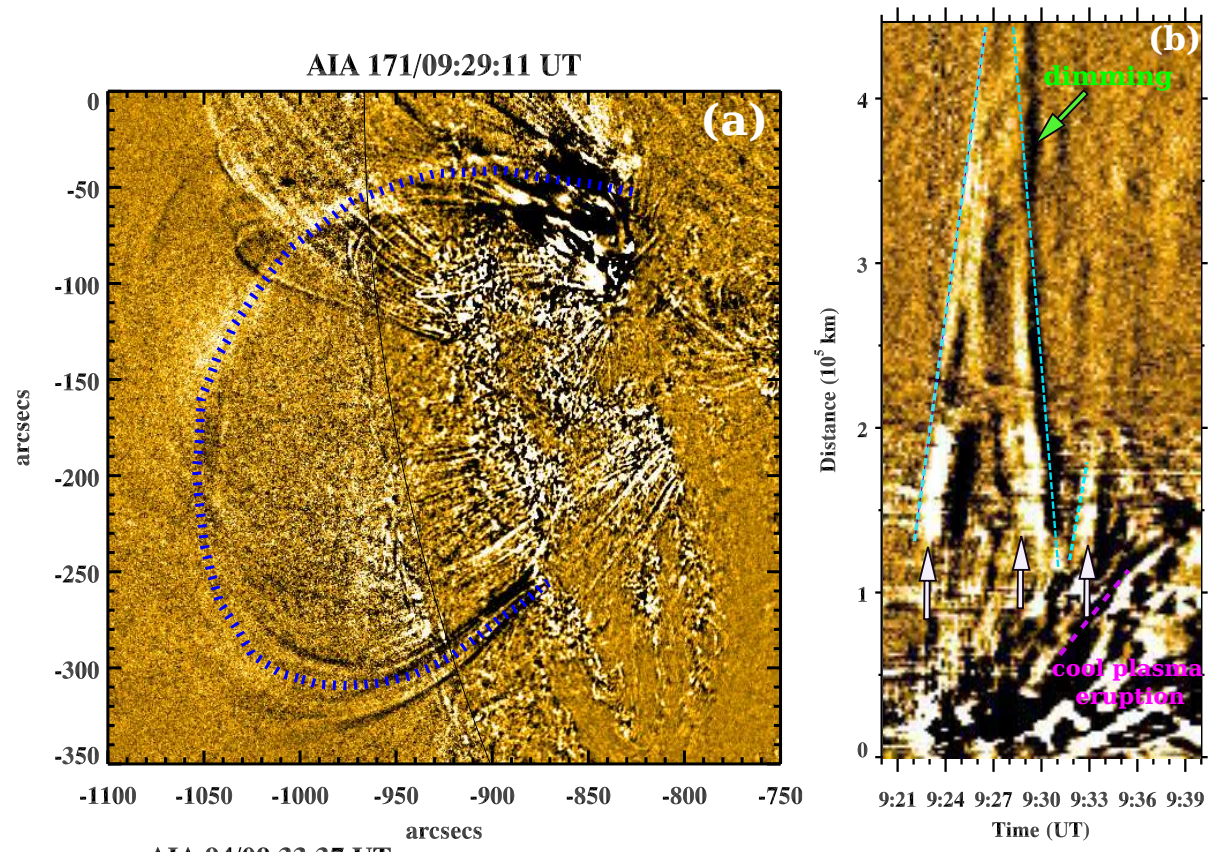

9:21 9:24 9:27 9:30 9:33 9:36 9:39 Time (UT)
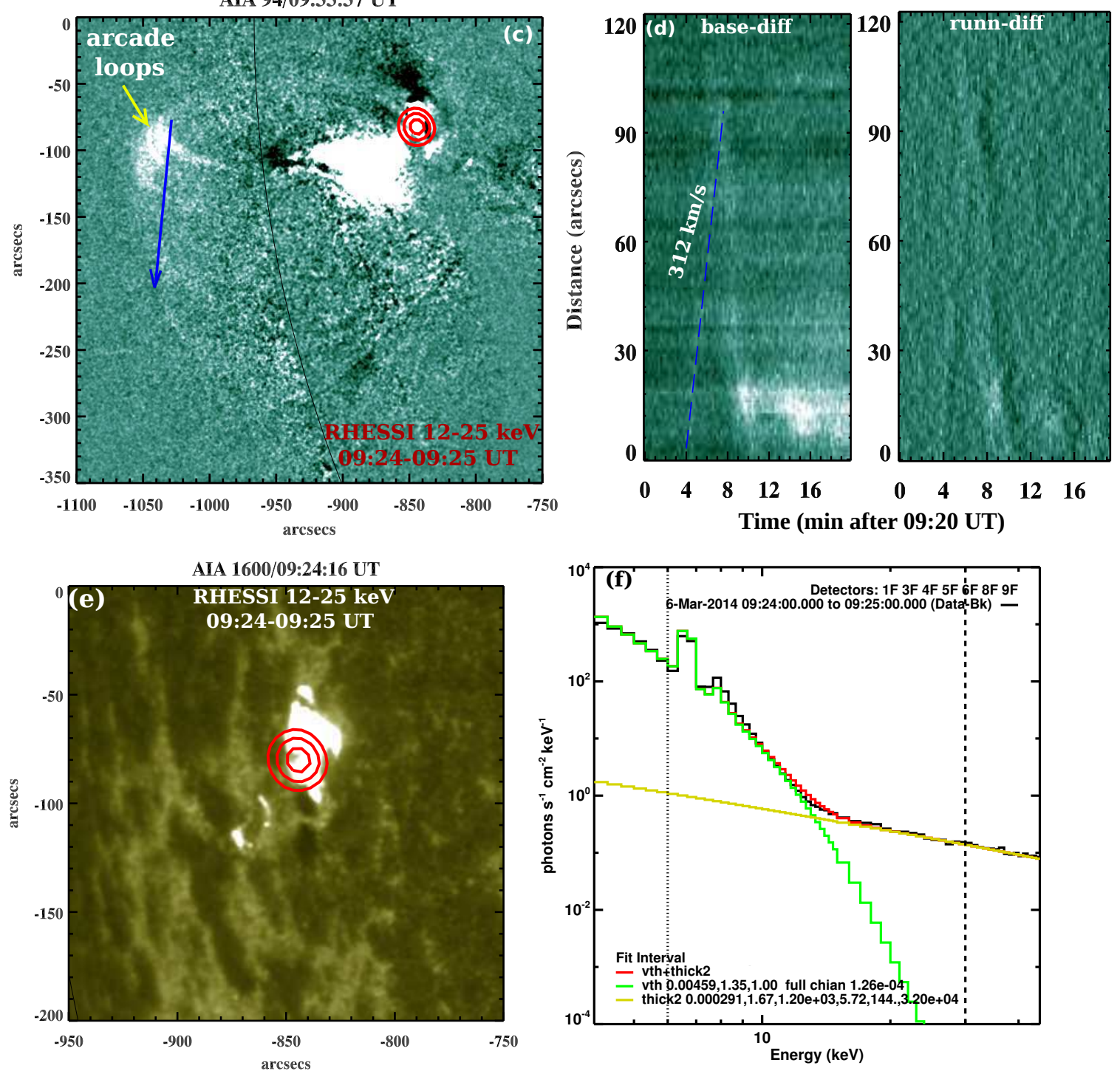

FIG. 3.- (a,c,e) AIA 171 and $94 \AA$ running and base difference images during the propagating disturbance along the loop system. RHESSI hard X-ray source (12-25 keV) overplotted on the AIA 94 and $1600 \AA$ images (contour levels: 50\%, 70\%, and 90\% of the peak intensity). (b,d) The temporal variation of the intensity along the selected path in 171 and $94 \AA$ images. (f) RHESSI X-ray spectrum fitted with an isothermal (green) and thick-target bremsstrahlung (yellow) components. (Animation is available.) 


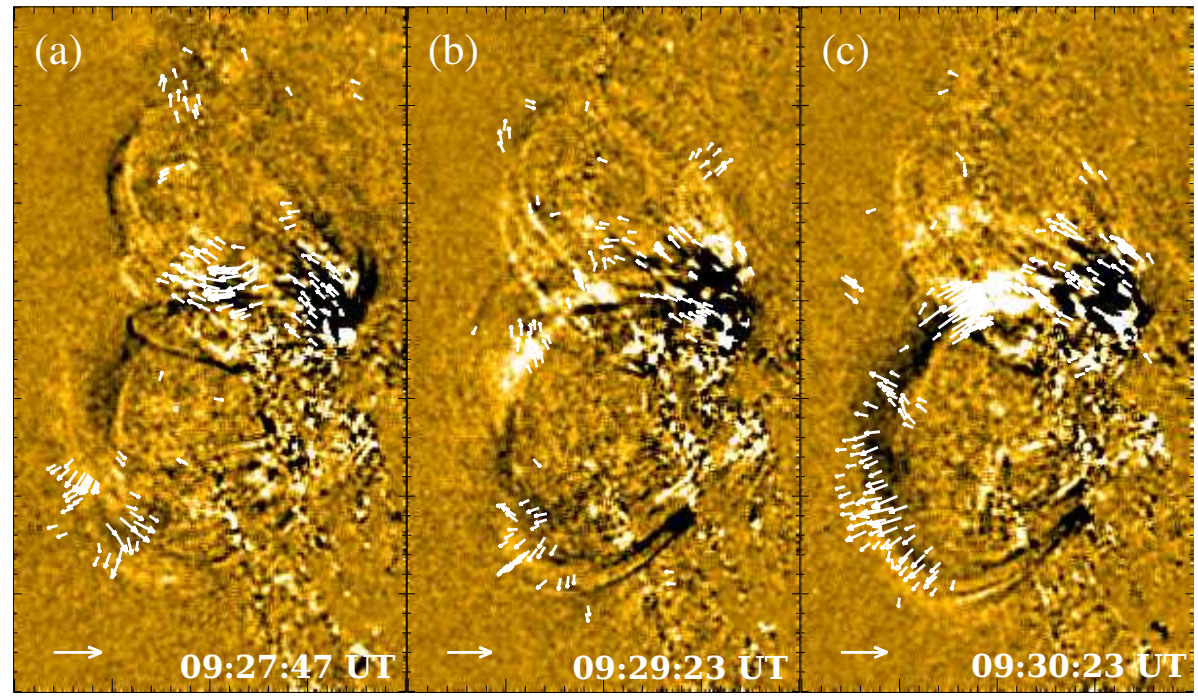

FIG. 4.- Flows around the flare site at three different phases: (a) outward propagation of the EUV wave; (b) backward propagation and onset of dimming; (c) the loops' upward expansion and motion back to the flare site. The length of the arrow in the bottom left represents $150 \mathrm{~km} \mathrm{~s}^{-1}$.
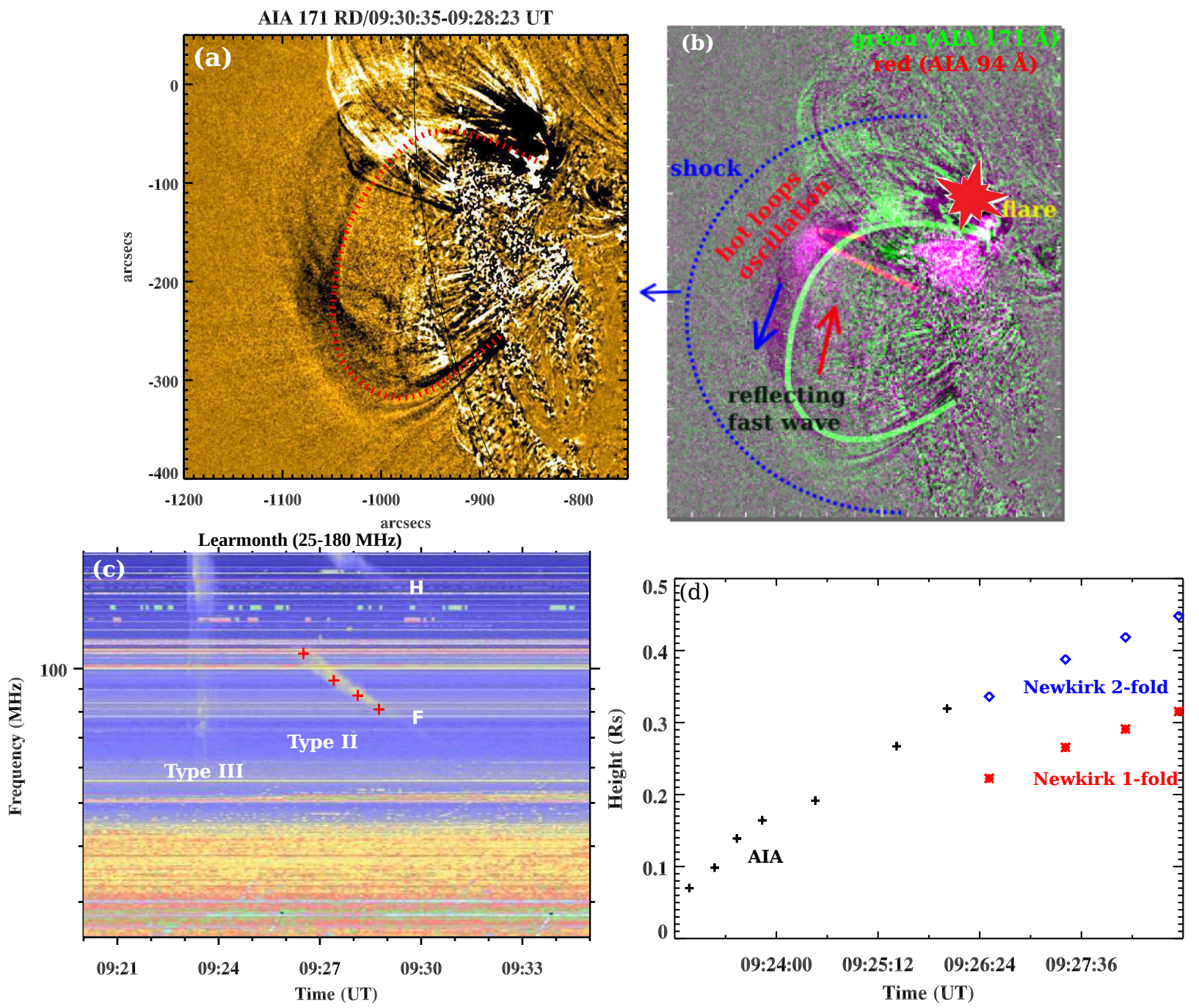

FIG. 5. - (a) Loop length estimation by curvature radius maximization method using AIA $171 \AA$ running difference image. (b) AIA 171 (green) and 94 (red) $\AA$ blended image with schematic cartoon over it. (c) Learmonth dynamic radio spectrum. F and $\mathrm{H}$ are the fundamental and second harmonic bands of the type II radio burst. (d) Shock height-time plot from the AIA $171 \AA$, Newkirk one-fold, and two-fold density models. 
used the optical flow code of Gissot \& Hochedez (2007) on the series of $171 \AA$ images. Figure 4 shows snapshots of the motions at three times. In Figure 4a, one sees propagation up from the flare site, a small region of outward motion to the north, as well as significant motion south along the $171 \AA$ loops. Figure $4 \mathrm{~b}$ shows the motion soon after the partial reflection, at the time of the onset of the dimming. Along the loop arcade, the motion is outward from the site of the dimming: both back toward the flare site and outward into the corona. The third image shows strong flows back to the flare site as well as extensive expansion of the loop system into the corona. Typically the highest plane-of-sky velocities are $\sim 150 \mathrm{~km} \mathrm{~s}^{-1}$ (the length of the arrows in the bottom-left corner). It is much less than the speed from the stack plot which measures the speed of faint fronts.

If we believe that the stack plot reveals the propagation of the wave front, then to deduce the phase speed of the wave, we need to estimate the loop length. STEREO images could be used to obtain the loop length with 3D reconstruction, but the footpoints of the arcade loops were not observed by STEREO because they were behind the western limb from STEREO. We used the curvature radius maximization method (Aschwanden 2009) to estimate the loop length. We reconstructed the $\mathrm{z}$ axis using this method, which provides a robust estimate of the 3D loop geometry from a single point observation. Figure 5a shows the best-fitted loop (red dotted curve). The estimated loop length is $\sim 600^{\prime \prime}$ and the travel time was $10 \mathrm{~min}$, so the phase speed of the wave $2 L / P \sim 1450 \mathrm{~km} \mathrm{~s}^{-1}$.

Figure 5b displays the blended AIA 171 (green) and 94 (red) A image, and a schematic cartoon over the image summarizing the whole activity. There was a partial AR filament eruption at 09:32 UT (marked by the dotted line), just after the returning wave reached the flare site. The AIA $171 \AA$ movie shows a surge-like ejection of filament material from the flare site. The EUV wave may play an important role in the triggering of the cool plasma ejection.

Figure 5c shows radio dynamic spectrum (25-180 MHz) from Learmonth radio observatory. We selected data points $(+)$ from the fundamental band (F) of type II radio burst to estimate the shock height using Newkirk onefold and two-fold density model (Newkirk 1961). Figure $5 \mathrm{~d}$ displays the shock height calculated from the AIA 171 $\AA$ channel $(+)$, Newkirk one-fold (blue star), and twofold (red diamond) density models. Note that AIA height is basically the projected height (in the sky-plane) of the shock front from the flare center. The estimated shock speeds from the Newkirk one- and two-fold density models are $556-451 \mathrm{~km} \mathrm{~s}^{-1}$ and $667-545 \mathrm{~km} \mathrm{~s}^{-1}$, respectively. These speeds are consistent with the decelerating shock speed from AIA. Moreover, the shock height is more consistent with the Newkirk two-fold density model.

\section{DISCUSSION AND CONCLUSION}

We report for the first time the propagation of a fastmode wave along and perpendicular to arcade loops, and its partial reflection from the opposite footpoint of the arcade loops (in $171 \AA$ and 193 channels). The phase speed of the longitudinal wave was $\sim 1450 \mathrm{~km} \mathrm{~s}^{-1}$.

The EUV wave caused swaying motion of an overlying arcade seen in AIA 94 and $131 \AA$. The initial plane-of-sky swaying velocity of the arcade was about $312 \mathrm{~km} \mathrm{~s}^{-1}$, and it was rapidly damped. Note that this speed is based on the initial amplitude of the hot loop oscillations, and is not the phase speed. Assuming the arcade consists of semi-circular loops with height $\sim 200^{\prime \prime}$ (Figure 3(c)), and a phase speed of the order of Alfvén speed, $\sim 1900 \mathrm{~km} \mathrm{~s}^{-1}$, the kink mode would have an oscillation period of about 8 min which is roughly the back-andforth period observed in Figure 3(d).

The nature of the observed fast MHD wave in closed arcade loops may be similar to the propagating fast-mode wave trains observed in the open-fan loops (Liu et al. 2011). Quasiperiodic wave trains are best observed in the AIA $171 \AA$ channel (Liu et al. 2011; Ofman et al. 2011; Kumar \& Manoharan 2013; Pascoe et al. 2013). However, here we observed only a single front (not wave trains) in the arcade loops and its close association with a flare. Theoretically, fast-mode waves can be guided by the magnetic field and trapped in a region of low Alfvénic speed (i.e., high density loops) (Roberts et al. 1984; Nakariakov \& Verwichte 2005).

Quasi-periodic wave trains are associated with quasiperiodicity of the flare (e.g., periodic reconnection). These signatures are generally observed in the hard Xray or AIA $1600 \AA$ channels (as a result of periodic particle acceleration). In our case, we do not see any quasi-periodic pulsation in the AIA $1600 \AA$ or hard Xray channels. Therefore, this event is related to a single burst of energy release that generates a single wave front.

The dome like expansion of a fast-mode wave was also observed in the AIA 193 and $211 \AA$ channels. The lateral expansion (in the southward direction) of the domeshaped wave coexists with the EUV wavefront propagating along the loops. The fast-mode wave propagates perpendicular and parallel to the magnetic field. Theoretically, the fast MHD wave can transport its energy in any direction (i.e., parallel or perpendicular to the magnetic field).

The fast-mode wave was closely associated with the compact flare. The EUV wave might be triggered either by the impulsive expansion of the flare loop or by thermal pressure (blast) generated during the flare energy release (Vršnak \& Cliver 2008; Kumar \& Innes 2013). In our case, we do not observe impulsive plasmoid ejection in either the cool or hot AIA channels before or during the onset of the EUV wave. Therefore, it is unlikely that the EUV wave observed here was triggered by the plasmoid ejection as reported in previous case studies (Klein et al. 1999; Kumar \& Manoharan 2013). There was no CME loop behind the EUV wave as observed in Veronig et al. (2010). Also we do not see two wave components (i.e., slow and fast), where the speed of the fast-mode wave (true shock wave) should be almost three times that of the slow wave (pseudo wave) (Chen et al. 2002; Chen \& Wu 2011; Kumar et al. 2013). The wave is most likely generated impulsively by the flare-related pressure pulse (or blast wave), and then propagated freely in the ambient medium. A metric type II radio burst also reveals the presence of a shock wave in the corona.

In conclusion, we presented the first observation of partial reflection and trapping of a fast magnetoacoustic wave in coronal arcade loops. Reflection and transmis- 
sion of shock waves from the boundary of a coronal hole (Gopalswamy et al. 2009; Olmedo et al. 2012) or from a different active region (Kumar \& Manoharan 2013) have been observed previously but, so far, there is no observational report of the reflecting and trapping of fast-mode waves in coronal arcade loops. When the fast-mode wave encounters a region of large gradient (from low to high) in the Alfvén speed, they may experience partial reflection. Thus a fast-mode wave traveling in a loop may be internally reflected at the loop edge and stay trapped in the loop. This observation also has an implication on the origin of the shock wave in the context of flares. We agree that most of the low coronal shocks are usually driven by the CME piston. However, here the observation of a re- flecting fast-mode wave in coronal loops strongly favours the flare origin (in this event) for the generation of the low coronal shocks rather than CMEs. Multiwavelength studies of similar events will be helpful to understand the excitation mechanisms of these waves in more detail.

We thank the referee for the positive and constructive comments/suggestions that improved the manuscript considerably. SDO is a mission for NASA's Living With a Star (LWS) program. RHESSI is a NASA Small Explorer. We would like to thank Prof. Valery M. Nakariakov, Hardi Peter, and Robert Cameron for several fruitful discussions.

\section{REFERENCES}

Asai, A., et al. 2012, ApJ, 745, L18

Aschwanden, M. J. 2009, Space Sci. Rev., 149, 31

Banerjee, D., Erdélyi, R., Oliver, R., \& O’Shea, E. 2007, Sol. Phys., 246, 3

Chen, P. F., Wu, S. T., Shibata, K., \& Fang, C. 2002, ApJ, 572, L99

Chen, P. F., \& Wu, Y. 2011, ApJ, 732, L20

De Moortel, I., \& Nakariakov, V. M. 2012, Royal Society of London Philosophical Transactions Series A, 370, 3193

Gissot, S. F., \& Hochedez, J.-F. 2007, A\&A, 464, 1107

Gopalswamy, N., et al. 2009, ApJ, 691, L123

Klein, K.-L., Khan, J. I., Vilmer, N., Delouis, J.-M., \& Aurass, H. 1999, A\&A, 346, L53

Kliem, B., Dammasch, I. E., Curdt, W., \& Wilhelm, K. 2002, ApJ, 568, L61

Kumar, P., Cho, K.-S., Chen, P. F., Bong, S.-C., \& Park, S.-H. 2013, Sol. Phys., 282, 523

Kumar, P., \& Innes, D. E. 2013, Sol. Phys., 288, 255

Kumar, P., Innes, D. E., \& Inhester, B. 2013, ApJ, 779, L7

Kumar, P., \& Manoharan, P. K. 2013, A\&A, 553, A109

Lemen, J. R., et al. 2012, Sol. Phys., 275, 17

Lin, R. P., et al. 2002, Sol. Phys., 210, 3

Liu, W., \& Ofman, L. 2014, Sol. Phys., 289, 3233

Liu, W., Title, A. M., Zhao, J., Ofman, L., Schrijver, C. J., Aschwanden, M. J., De Pontieu, B., \& Tarbell, T. D. 2011, ApJ, 736, L13

Ma, S., Raymond, J. C., Golub, L., Lin, J., Chen, H., Grigis, P., Testa, P., \& Long, D. 2011, ApJ, 738, 160
Nakariakov, V. M., \& Verwichte, E. 2005, Living Reviews in Solar Physics, 2, 3

Newkirk, G., Jr. 1961, ApJ, 133, 983

Nitta, N. V., Aschwanden, M. J., Freeland, S. L., Lemen, J. R., Wülser, J.-P., \& Zarro, D. M. 2014, Sol. Phys., 289, 1257

Ofman, L., Liu, W., Title, A., \& Aschwanden, M. 2011, ApJ, 740, L33

Ofman, L., \& Wang, T. J. 2008, A\&A, 482, L9

Olmedo, O., Vourlidas, A., Zhang, J., \& Cheng, X. 2012, ApJ, 756,143

Patsourakos, S., \& Vourlidas, A. 2012, Sol. Phys., 281, 187

Pascoe, D. J., Nakariakov, V. M., \& Kupriyanova, E. G. 2013, A\&A, 560, AA97

Roberts, B., Edwin, P. M., \& Benz, A. O. 1984, ApJ, 279, 857

Schou, J., et al. 2012, Sol. Phys., 275, 229

Shen, Y., \& Liu, Y. 2012, ApJ, 753, 53

Thompson, B. J., et al. 1999, ApJ, 517, L151

Uchida, Y. 1970, PASJ, 22, 341

Veronig, A. M., Muhr, N., Kienreich, I. W., Temmer, M., \& Vršnak, B. 2010, ApJ, 716, L57

Vršnak, B., \& Cliver, E. W. 2008, Sol. Phys., 253, 215

Wang, T., Solanki, S. K., Curdt, W., Innes, D. E., \& Dammasch, I. E. 2002, ApJ, 574, L101

Wang, T. J., Solanki, S. K., Curdt, W., Innes, D. E., Dammasch, I. E., \& Kliem, B. 2003a, A\&A, 406, 1105

Wang, T. J., Solanki, S. K., Innes, D. E., Curdt, W., \& Marsch, E. 2003b, A\&A, 402, L17 